swept, silica-rich dust. The effects on respiratory heath consequent on this exposure are largely unknown.

Objective To examine associations between environmental GMWD dust exposure and respiratory health effects in adults. Methods This was a cross-sectional study of 93, 133 and 84 people in high (home $<500 \mathrm{~m}$ from GMWD), moderate $(500 \mathrm{~m}-1 \mathrm{~km})$ and low $(>20 \mathrm{~km})$ exposure groups respectively. We calculated a cumulative exposure index (CEI) based on exposure groups and years of residence. Participants were interviewed for respiratory symptoms, had chest X-rays (read by three experienced readers for tuberculosis and silicosis) and spirometry. We used multivariate logistic regression to examine the effect of dust exposure on respiratory health, and multiple linear regression to determine if CEI was associated with $\%$ predicted FEV1 and FVC. We adjusted for socioeconomic status, smoking and occupational and biomass fuel exposure.

Results Being in the high exposure group versus the low was associated with elevated adjusted odds ratios (aORs) for upper respiratory (aOR: 2.76, 95\% CI: 1.28-5.97) and ocular symptoms (aOR: 4.68; 95\% CI: 1.87-11.68), chest wheezing (aOR: 3.78; 95\% CI: 1.60-8.96) and spirometrydiagnosed COPD (aOR: 8.17; 95\% CI: 1.01-65.85). We found similar associations for the high versus medium exposure groups, but no significant associations in the medium relative to the low group. Exposure had no significant effect on the risks of chronic bronchitis and tuberculosis. We found similar significant results for CEI as for the exposure groups analyses. On linear regression, CEI was not associated with $\%$ predicted FEV1, but, surprisingly, FVC was positively associated with CEI. No participant had radiological features of silicosis.

Conclusion Residents residing $<500 \mathrm{~m}$ from GMWDs had elevated aORs for respiratory health effects. Both exposure measures (exposure groups and CEI) yielded similar results.

\section{A.6 RECENT ORGANIC DUST EXPOSURE AND PROGNOSIS OF ASTHMA AND CHRONIC OBSTRUCTIVE LUNG DISEASE (COPD). A NATIONWIDE REGISTER BASED FOLLOW-UP STUDY}

\begin{abstract}
${ }^{1,2}$ Anne Vested*, ${ }^{3}$ Ioannis Basinas, ${ }^{1,4}$ Alex Burdorf, ${ }^{1}$ Grethe Elholm, ${ }^{5}$ Dick Heederik, ${ }^{6}$ Gitte Jacobsen, ${ }^{2}$ Henrik Kolstad, ${ }^{5}$ Hans Kromhout, ${ }^{7}$ Øyvind Omland, ${ }^{1}$ Torben Sigsgaard, ${ }^{2}$ Jesper Medom Vestergaard, ${ }^{5}$ Inge Wouters, ${ }^{1,8}$ Vivi Schlünssen*. ${ }^{1}$ Department of Public Health, Danish Ramazzini Centre, Aarhus University, Aarhus, Denmark; ${ }^{2}$ National Research Center for the Working Environment, Copenhagen, Denmark; ${ }^{3}$ Department of Occupational Medicine, Danish Ramazzini Centre; Aarhus University Hospital, Aarhus, Denmark; ${ }^{4}$ Institute of Occupational Medicine (IOM), Edinbourgh, UK; ${ }^{5}$ Erasmus MC, Department of Public Health, Rotterdam, the Netherlands; ${ }^{6}$ Utrecht University, Institute for Risk Assessment Sciences, Utrecht, the Netherlands; ${ }^{7}$ Regional Hospital West, Department of Occupational Medicine, Danish Ramazzini Centre, Herning, Denmark; ${ }^{8}$ Aalborg Universitetshospital, Department of Occupational Medicine, Danish Ramazzini Centre, Aalborg, Denmark
\end{abstract}

\subsection{6/OEM-2019-EPI.61}

Background Air pollutants at work can contribute to onset of asthma and COPD. How occupational air pollutants affect the prognosis of asthma or COPD among exposed workers is not well established.

Objective We aimed, among individuals with a hospital diagnosis of asthma or COPD, to study the association between recent exposure to organic dust, and hospital readmission and overall mortality.
Methods The study population comprised individuals ever employed in farming or wood industry with asthma $(n=4002)$ or COPD $(n=2429)$ identified in the Danish national patient register of individuals born 1933-1977. Subjects were included the year immediately following their first asthma or COPD hospital contact (earliest in 1998) and followed until first asthma or COPD readmission, death, or December 31 st 2007. Exposure data was obtained through register-based industry codes from 1997-2007 combined with time-dependent farming and wood industry-specific exposure matrices. We used logistic regression analysis with discrete survival function adjusted for age, calendar year, sex, mineral dust exposure, socioeconomic status, and labour-force participation.

Results Among individuals with asthma, the risk of hospital readmission was slightly increased among the exposed vs. the non-exposed, RRadj 1.17 (0.91-1.50), but with no exposure trend. A non-significant decrease in mortality was seen for organic dust exposure and mortality for those individuals, RRadj 0.71 (0.24-2.06).

The risk of a COPD readmission among individuals with COPD was decreased among exposed vs. non-exposed individuals, RRadj 0.67 (0.46-0.98), but with no exposure trend. Mortality was non-significantly increased for exposed vs. non-exposed individuals with COPD, RRadj $1.59 \quad(0.82-$ 3.08).

Conclusion We did not observe significant associations between recent exposure to organic dust and readmission for COPD/asthma or overall mortality except for a decreased risk for COPD readmission. Selection effects are presumably playing a role. We did adjust for socioeconomic position and labour-force participation but not for smoking which is a limitation.

\section{Burden of Occupational Disease and Injury}

\section{O3B.1 BURDEN OF WORK ABSENCE DUE TO COMPENSABLE ROAD TRAFFIC CRASHES IN VICTORIA, AUSTRALIA}

Shannon Gray*, Alex Collie. Insurance Work and Health Group, School of Public Health and Preventive Medicine, Monash University, Melbourne, Australia

\subsection{6/OEM-2019-EPI.62}

Background The burden of road traffic crashes (RTC) is commonly reported using fatalities and hospital admissions. Disruption to regular activities, such as work, is rarely reported, yet known to have significant economic and human costs. In the state of Victoria, Australia, people injured and unable to work due to RTC may have treatment and income support provided either through the RTC compensation system or through workers' compensation. By examining data from both systems, this study sought to determine the rate (per 100000 working population) of RTC injury resulting in work absence, and to quantify the amount of working time lost to RTC injury.

Methods Data from each compensation system were harmonised. Analysis included claims from RTCs that occurred between July 12003 and June 302013 by 15-65 year olds who received at least one day of income support. Fatalities and rejected claims were excluded. Time lost was calculated as the total weeks of income support. Non-parametric tests were 
performed to determine differences between exposure variables (e.g. male versus female).

Results There were 36640 workers meeting inclusion criteria (average 305 cases per month; mean rate per month of 11.6 per 100000 working population). A total of 1,121,863 lost working weeks were compensated, with a median of 10 weeks. Thirty-two percent of cases did not have a record of attending hospital. The lowest median duration of time loss was among those involved in a train or tram crash (2.9 weeks) and the highest among those with quadriplegia (142.2 weeks). Duration of income support was significantly different within age, sex, injury type, severity, crash type, and compensation system groups.

Conclusions Results showed that RTC injuries of all severities caused considerable work absence. Measures of work absence can complement existing measures of RTC burden.

\section{O3B.2 OCCUPATIONAL EXPOSURES AND SICKNESS ABSENCE TRAJECTORIES AMONG FINNISH EMPLOYEES: A REGISTER LINKED NATIONALLY REPRESENTATIVE FOLLOW-UP STUDY}

\begin{abstract}
1,2Tea Lallukka*, 'Leena Kaila-Kangas, ${ }^{2,3}$ Minna Mänty, ${ }^{4}$ Johanna Seppänen, ${ }^{1}$ Eija Haukka, ${ }^{1}$ Johanna Kausto, ${ }^{1}$ Päivi Leino-Arjas, ${ }^{4}$ Risto Kaikkonen, ${ }^{1} J a a n a ~ H a l o n e n, ~ 1,2$ Rahman Shiri. ${ }^{1}$ Finnish Institute of Occupational Health, Helsinki, Finland; ${ }^{2}$ Department of Public Health, University of Helsinki, Helsinki, Finland; ${ }^{3}$ Department of Research, Development and Innovation (RDI), Laurea University of Applied Sciences, Vantaa, Finland; ${ }^{4}$ National Institute for Health and Welfare, Helsinki, Finland
\end{abstract}

\subsection{6/OEM-2019-EPI.63}

Background Physical workload factors have been linked to the risk of sickness absence, however, some work exposures can also be protective of work ability. We aimed to first identify sickness absence trajectories in a nationally representative occupational cohort, and second to examine the associations between physical workload factors, potential protective factors and sickness absence trajectories.

Methods We examined a nationally representative cohort of working aged Finnish adults interviewed and medically examined at baseline in 2000-2001 $(n=3814)$. Both potentially protective (sitting and keyboard use) and risk factors (e.g. handling of heavy loads and vibration) were included. All medically confirmed sickness absence periods 2002-2008 were included in the trajectory analyses to identify latent groups with similar sickness absence trends. These data were derived from the registers of the Social Insurance Institution of Finland. The models were adjusted for sociodemographic factors, health behaviors and physical and mental health.

Results We found a group with a very low level, a group with a stable low level, and a group with a high and increasing level of sickness absence. After full adjustments, prolonged sitting and use of keyboard emerged as protective factors that were associated with a lower risk of belonging to the trajectory with high and increasing sickness absence (HR 0.60; 95\% CI 0.45-0.80). In turn, the risk increased with the number of physical workload factors reported, and was highest for those with four or more workload factors (HR 2.84; 95\% 2.08-3.88).

Conclusions Physical work is associated with the risk of long-term sickness absence, with the highest risk found for those with several different physical workload factors. As the risk remained after controlling for various pertinent risk factors, this highlights the need to find ways to better maintain work ability of those with the physically most strenuous work.

\section{B.3 ESTIMATING THE IMPACT OF CHANGES TO OCCUPATIONAL STANDARDS FOR SILICA EXPOSURE ON LUNG CANCER MORTALITY}

${ }^{1}$ Alex Keil, 'David Richardson, ${ }^{1}$ Daniel Westreich, ${ }^{2}$ Kyle Steenland ${ }^{*} .{ }^{1}$ Rollins School of Public Health, Emory U, Atlanta, USA; ${ }^{2}$ Dept Epidemiology, UNC, Chapel Hill, US

\subsection{6/OEM-2019-EPI.64}

Background Respiratory exposure to silica is associated with the risk of death due to malignant and non-malignant disease. 2.3 million U.S. workers are exposed to silica. Occupational exposure limits for silica are derived from a number of lines of evidence, including observational studies. Observational studies may be subject to healthy worker survivor bias, which could result in underestimates of silica's impact on worker mortality and, in turn, bias risk estimates for occupational exposure limits.

Methods Using data on 65999 workers pooled across multiple industries, we estimate the impacts of several hypothetical occupational exposure limits on silica exposure on lung cancer and all-cause mortality. We use the parametric g-formula, which can account for healthy worker survivor bias.

Results Assuming we could eliminate occupational exposure, we estimate that there would be 20.7 fewer deaths per 1000 workers in our pooled study by age 80 (95\% confidence interval: 14.5, 26.8), including 3.91 fewer deaths due to lung cancer (95\% CI: 1.53, 6.30). Less restrictive interventions demonstrated smaller, but still substantial risk reductions.

Conclusions Our results suggest that occupational exposure limits for silica can be further strengthened to reduce silicaassociated mortality and illustrate how current risk analysis for occupational limits can be improved.

\section{O3B.5 THE MENTAL HEALTH OF POLICE, FIREFIGHTERS AND PARAMEDICS IN THE UK BIOBANK: A COMPARISON WITH THE GENERAL WORKING POPULATION}

Sharon Stevelink*, Nicola Fear, Matthew Hotopf. King's College London, London, UK

\subsection{6/OEM-2019-EPI.65}

It is our responsibility to protect and look after the health of members of the emergency services as this directly impacts on the country's readiness to respond to these disasters and is a critical part of our duty of care towards this important group of workers. This study examined the mental health outcomes and associations with individual and job characteristics among emergency services personnel compared to a random sample of working people, thereby using data from the UK Biobank. This data source contains data on over half a million adults in the UK, who were at the time of recruitment between 40 69 years. Over 280000 reported being in work. Current emergency services personnel were identified based on Standard Occupational Classification (SOC) 2000 codes. A random sample of age and sex matched people working in other occupations were selected from the UK Biobank for comparative purposes. The prevalence of the outcomes of interest, based 